\title{
SESIÓN TEMÁTICA I: Continuidad y Transversalidad en Educación Médica: El Paradigma de la Medicina de Familia
}

\section{Moderadora: Gloria Guerra de la Torre}

\section{Jueves 27 de octubre - 17.30 horas Salón de Actos del Vicerectorado}

\author{
Desde los inicios: Participación de la Medicina \\ de Familia en la docencia Pregrado \\ en la Unión Europea \\ Pablo Bonal Pitz \\ Coordinador del Grupo de Pregrado de SEMFyC. \\ Facultad de Medicina. Universidad de Sevilla
}

En 1910 la medicina general comenzó a alejarse de las Facultades de Medicina (FM) a consecuencia del Informe Flexner ${ }^{1}$. Posteriormente, con la eclosión de las especialidades hospitalarias y de la biomedicina y se ahondo el abismo entre las FM y los Médicos de Familia (MF). Los estudiantes perdieron de la perspectiva global de su profesión, la visón de la historia natural de la enfermedad, la percepción de los problemas de salud en el entorno familiar y social, y su prevalencia real, la integración de la dimensión psíquica, física y social del paciente, la promoción de la salud y la prevención de la enfermedad en la actividad clínica diaria, etc. Desde los años 60, diversos países occidentales se replantearon este tipo de docencia exclusivamente hospitalaria de la medicina e incorporaron la MF a las FM como materias obligatorias y con Departamentos universitarios de $\mathrm{MF}^{2-4}$ (Tabla 1). Actualmente, la MF incorpora en las FM europeas un contacto idóneo de los alumnos con los pacientes en su entorno familiar y comunitario para el aprendizaje de la medicina clínica y comunitaria, una red asistencial en Atención Primaria (AP) con una gran diversidad de problemas de salud prevalentes en la población, sanos y enfermos en situaciones clínicas ideales para el aprendizaje de la medicina, médicos asistenciales y docentes (más del 50\% del total). Además, en las FM se prevén cambios docentes en los hospitales de tipo cuantitativos (quiere menos alumnos y necesita más residentes) y cualitativos (tienen menos pacientes hospitalizados por menores estancias medias y en situaciones clínicas más complejas, graves, de baja prevalencia y con necesidad de muy alta tecnología). La AP dispone de la mayor red docente y asistencial de los servicios de salud con recursos muy cualificados para hacer docencia idónea en pregrado, postgrado y continuada. Por lo anterior, la MF en Europa juega un papel importante en todo el proceso formativo de los futuros médicos. Facilita una formación más orientada y adaptada a las necesidades sociales y sanitarias actuales, y aporta un incremento de la elección vocacional para trabajar en AP, donde la OMS determina que 6 de cada 10 estudiantes ejercerán como MF. Sin embargo, en España ese casi $60 \%$ no habrá recibido ningún tipo de formación al respecto. La universidad española debe normalizarse con respecto a Europa, sobre todo en el actual proceso de adecuación y convergencia del EEES.

Tabla 1. Presencia de la Medicina de Familia en las Universidades Europeas

\begin{tabular}{|c|c|c|}
\hline País & $\begin{array}{l}\text { Asignatura } \\
\text { en Cursos }\end{array}$ & $\begin{array}{c}\text { \% Facultades } \\
\text { con Dpto. } \\
\text { de Medicina Familia }\end{array}$ \\
\hline Alemania & $4^{\circ}, 5^{\circ}$ y $6^{\circ}$ & 100 \\
\hline Austria & $11^{\circ}$ y $12^{\circ}$ semestre & 50 \\
\hline Bélgica & $4^{\circ}$ & 100 \\
\hline Croacia & Periodo clínico & 100 \\
\hline Dinamarca & Periodo clínico & 100 \\
\hline Eslovenia & $6^{\circ}$ & 100 \\
\hline España & $6^{\circ}$ en 3 Facultades & 0 \\
\hline Finlandia & $1^{\circ}, 3^{\circ}, 4^{\circ}$ y $6^{\circ}$ & 100 \\
\hline Francia & Periodo clínico & 30 \\
\hline Grecia & $6^{\circ}$ & 20 \\
\hline Holanda & Periodo clínico & 100 \\
\hline Hungría & $5^{\circ}$ y $6^{\circ}$ & 75 \\
\hline Irlanda & $4^{\circ}, 5^{\circ}$ y $6^{\circ}$ & 100 \\
\hline Italia & $6^{\circ}$ en 4 Facultades & 0 \\
\hline Malta & $3^{\circ}, 4^{\circ}$ y $5^{\circ}$ & 100 \\
\hline Noruega & $10^{\circ}$ Rotación & 100 \\
\hline Polonia & $6^{\circ}$ & 100 \\
\hline Portugal & $6^{\circ}$ & 100 \\
\hline R. Unido & Periodo clínico & 100 \\
\hline Suecia & $6^{\circ}, 7^{\circ}, 8^{\circ}$ y $9^{\circ}$ semestre & 100 \\
\hline
\end{tabular}

Bibliografía:

1. Flexner A. Medical Education in the United States and Canada. A report to the Carnegie Foundation for the advancement of Teaching. Bulletin No. 4. Boston, Massachusetts: Updyke; 1910.

2. Fraser RC, Preston-Whyte E. The contribution of Academic General Practice to Undergraduate Medical Education. The Royal College of General Practitioners. Londres 1988.

3. Howie JG, Hannay DR. General Practice in the Medical Schools of the United Kindong. The Mackenzie Report. Mackenzie Fund. Londres 1986.

4. Jonas HS, Etzel SI, Barzansky B. Educational Programs in US Medical Schools. JAMA 1991; 266: 913-920.

\section{Innovaciones en las metodologías y contenidos: Programa de la Especialidad de Medicina de Familia y Comunitaria en España Verónica Casado Vicente. Comisión Nacional de la Especialidad de MFyC. Facultad de Medicina. Universidad de Valladolid}

El éxito de la Atención Primaria depende de que la Sociedad acepte su importancia y de que los medios para su mejora formen parte esencial de la política de formación y prestación de servicios de Atención Primaria. Bárbara Starfield

El programa de Medicina Familiar y Comunitaria fue aprobado en BOE el 3 de mayo de 2005 pero en el análisis de situación, diseño, elaboración, consenso y aprobación se han 
invertido más de 5 años. Las claves de este programa pivotan sobre cuatro grandes ejes:

- La adaptación al perfil profesional. Ha contribuido a definir y delimitar el cuerpo doctrinal de la Medicina de Familia que a lo largo de los años ha pasado de ser un área indiferenciada (medicina general) a una especialidad con campo de actuación propio, enfoque y paradigma definido y contenidos claros. El programa define los niveles de responsabilidad en el que se determinan los límites competenciales del médico de familia fundamentales para las áreas transversales.

- La adaptación a los valores profesionales. Se incorpora la formación en valores profesionales, en un momento en que los valores negativos están muy presentes en las realidades profesionales (burn-out, medicina defensiva...). Lo que da consistencia a todas las competencias de un médico de familia tiene mucho que ver con sus valores profesionales y estos se enmarcan con sus compromisos: con las personas y su salud, con la sociedad, con la mejora continua y el compromiso científico, el compromiso ético y el compromiso con la propia especialidad y con la formación.

- La flexibilidad basando la formación en la individualización de la misma con un incremento progresivo de la responsabilidad y de la personalización. Este hecho fuerza la incorporación de metodologías educativas que pivotan en el "aprender a aprender" y "centrado en el que aprende". Se incorporan dos metodologías básicas que son el autoaprendizaje dirigido y el aprendizaje de campo y se potencia los métodos de evaluación formativa que permiten la tutorización estructurada y continua. Su eje fundamental es la relación tutor-residente. Se complementa el programa con al menos 200 horas lectivas teórico-prácticas que se basan en cursos para el aprendizaje de contenidos nuevos, en talleres para el aprendizaje de habilidades y en trabajo en grupo para el aprendizaje de actitudes. El programa incorpora los niveles de prioridad que define en qué debe ser competente un médico de familia para ser apto, para ser destacado y para ser excelente.

- La pluripotencialidad: el programa se dirige no sólo a la formación de médicos de familia que vayan a desarrollar su actividad en un centro de salud, sino que permite servir de base formativa para la posterior formación en otras áreas de capacitación o para desempeñar su trabajo en otros ámbitos laborales y para el desarrollo de la troncalidad.

- El tiempo: Una vez incorporadas las nuevas áreas de formación (comunicación, gestión clínica, bioética, razonamiento clínico, atención a las personas en riesgo social, inmigrantes, violencia doméstica, atención a los adolescentes, atención a las personas con consumo de sustancias de riesgo...) y analizados los tiempos mínimos de aprendizaje para garantizar la adquisición de las competencias se ha incrementado el tiempo a 4 años. Bibliografía:

1. Comisión Nacional de Medicina Familiar y Comunitaria. Guía de Formación de Especialistas: Medicina Familiar y Comunitaria. Madrid 2005.

http://www.mcs.es/profesional/formacionSanitaria

Especializada/guiasFormacion/pdf/medifamiliar.pdf

\author{
Evaluación de programas de Postgrado: \\ La ECOE de Medicina de Familia \\ José $\mathrm{M}^{\mathrm{a}}$ Cots Yago \\ Coordinador de Docencia. ICS. \\ Divisió d'Atenció Primària. \\ Facultad de Medicina. Universidad de Barcelona
}

En toda profesión, incluida la medicina de familia, determinar que competencias son asumidas es una tarea básica para los profesionales. En nuestra especialidad tenemos diferentes documentos, tanto a nivel nacional como internacional, donde se especifican las competencias del médico de familia y en concreto es el Programa de la Especialidad de Medicina Familiar y Comunitaria de la Comisión Nacional de la especialidad donde encontramos de forma explicita dichas competencias. En el se determinan las diferentes áreas que debemos ser competentes: atención al individuo, familia y comunidad, tanto en actividades preventivas como curativas o de rehabilitación, así como en las áreas docente e investigadora. El modelo ideal de médico competente debe tener un equilibrio entre conocimientos teóricos, capacidad para aplicarlos y actitudes humanas.

En los programas de evaluación de la competencia tenemos dos dimensiones: en primer lugar debemos saber que componentes de la práctica profesional debemos evaluar y como deben ser medidos y en segundo lugar cuales son los estándares deseados. En el momento que queramos responder a estas preguntas debemos valorar cuando y como introduciremos la evaluación de la competencia profesional. Evaluar la competencia no es fácil, en primer lugar porque en nuestra cultura el hecho de ser evaluados se identifica con un sentido peyorativo, en segundo lugar porque el sentirse observado es vivido como algo que descubre interioridades, y por último el hecho de detectar déficits competenciales comportará necesariamente buscar soluciones formativas.

¿Cómo evaluaremos la competencia en Medicina de Familia?: En sentido estricto no existe la competencia como un todo sino que es el conjunto de diferentes elementos. Para evaluar la competencia no tenemos un instrumento único para su medición sino que deberemos utilizar un conjunto de instrumentos. Así a modo de ejemplo es conocido que uno de los instrumentos más usados para evaluar la competencia son las pruebas de Evaluación Clínica Objetiva y Estructurada (ECOE) en las cuales se evalúa una parte de las competencias como son la práctica clínica, así deberemos combinarlo con otros instrumentos para llegar a evaluar la competencia profesional. Existen diferentes métodos que utilizan combinaciones de instrumentos evaluativos, todos con una eficacia demostrada pero también con sus limitaciones. Entre ellos tenemos los métodos indirectos como los exámenes escritos, tipo test o preguntas abiertas de respuesta corta, los exámenes orales estructurados a partir de casos clínicos, los pacientes estandarizados o las simulaciones con maniquís u ordenadores. Estos instrumentos evalúan desde conocimientos (saber), competencia (saber como) o actuación (demostrar como). Los méto- 
dos directos como auditorias clínicas, video grabaciones $\mathrm{u}$ observación de la práctica evalúan la práctica real (hacer).

¿Qué componentes deberemos evaluar?: La especialidad de Medicina Familia y Comunitaria abarca un amplio campo de conocimientos y habilidades. Esto viene determinado porque nuestra especialidad tiene un sentido horizontal, conteniendo muchos conceptos de otras especialidades verticales. Definir el corpus de nuestra especialidad es importante porque si quisiéramos evaluar todos los componentes serios un ejercicio poco eficaz, así se definirán aquellas competencias básicas e indispensables para el ejercicio de la medicina de familia. El estándar de la evaluación puede colocarse al nivel de exigencia que se desee pero siempre de forma predeterminada. En estas pruebas, en general, se coloca a un nivel de corte de suficiencia, asegurando que el médico que esta por encima del corte es competente y aquel que no supera el corte es incompetente. Esto quiere decir que las pruebas no discriminan entre mas competente, de los competentes, o mas incompetente, de los incompetentes.

¿Por qué evaluar la competencia de los médicos de familia?: En diferentes campos, y en el docente es un vivo ejemplo, los médicos de familia hemos sido pioneros a la hora de desarrollar y poner en práctica modelos formativos innovadores. Es destacado y conocido la implantación de nuestro programa de la especialidad, la tutorización y evaluación de la formación postgraduada, los planes de formación continuada o la acreditación de actividades de formación. En esta línea la evaluación de la competencia tiene que ser un terreno en el que los médicos de familia iniciemos sigamos siendo pioneros no solo en introducirlo sino también en darle continuidad y expertez. Bibliografia:

1. Epstein RM, Hundert E. Defining and assessing professional competence. JAMA 2002; 287: 226-235

2. 2-Sociedad Española de Medicina de Familia y Comuni-taria. Perfil profesional básico del médico de familia. Documentos semfyc $n^{a} 4$

3. Sociedad Española de Medicina de Familia y Comunitaria. Evaluación de la competencia: reto o necesidad. Docu-mentos semfyc $n^{\mathrm{a}} 18$

4. Ruiz E, Florensa E, Cots JM, Sellalrès J, Iruela a, Blay C et al. Primeras experiencias en evaluación de la competencia clínica de los médicos de familia de Catalunya. Aten Primaria 2001, 28:105-109.

5. Ely JW; Dawson JD, Young PR. Malpractice claims against family physicians: are the best doctor sued more?. J Fam Pract 1999; 48:23-30.

\section{Vertebración de la Educación Médica Continuada. El Portafolio de Medicina de Familia. \\ José Antonio Prados Castillejo \\ Coordinador de Área del Portafolio \\ Evaluativo Estandarizado de la SEMFyC. \\ Escuela Andaluza de Salud Pública}

La calidad en la actividad asistencial es una necesidad y un deber de todo profesional sanitario. Es muy difícil progresar como profesional, realizar un correcto desarrollo profesional y por tanto formarse de forma adecuada sin una herramienta que objetive tus competencias e incompetencias conscientes y que a la vez identifique competencias e incompetencias inconscientes. Pero este feed-back para profesionales que ya desarrollan una labor, no puede estar basado solo en lo que un profesional demuestra en situación de laboratorio. Es decir, no es suficiente con demostrar lo que yo soy capaz de hacer sino que la información puede ser mas útil si habla de lo que en realidad estoy o no haciendo con mis pacientes. Los sistemas de recertificación y acreditación por tanto deberían basarse en gran parte en evaluaciones de la competencia en el cuarto escalón de Miller (práctica real). Por otro lado, todo sistema de evaluación debe tener como objetivo la formación (y no viceversa) por lo que el impacto educativo de la propia herramienta debe ser una prioridad a la hora de diseñar sistemas de evaluación. Con estos dos objetivos fundamentalmente, surge un circuito de evaluación denominado Portafolio. Consiste en una recopilación de documentos (en papel, audio o videograbación) con los que el profesional demuestra su desarrollo profesional en base a la existencia en los mismos de evidencias de que un determinado nivel de competencia existía previamente o se ha alcanzado durante el propio proceso de evaluación. Utiliza habitualmente las siguientes fuentes de evidencias:

\begin{tabular}{|l|l|}
\hline $\begin{array}{l}\text { Registros clínicos } \\
\text { (Historia, informes de derivación...) }\end{array}$ & Tutorías y planes docentes \\
\hline $\begin{array}{l}\text { Plan de aprendizaje } \\
\text { (evidencias de aprendizaje } \\
\text { en proceso o finalizado) }\end{array}$ & $\begin{array}{l}\text { Diario de reflexión } \\
\text { (emociones y sentimientos } \\
\text { generados por el aprendizaje) }\end{array}$ \\
\hline Audio y Videograbaciones & Datos curriculares \\
\hline Incidentes críticos & Auditoria, Autoauditorias \\
\hline $\begin{array}{l}\text { Indicadores de la práctica } \\
\text { perfil farmacológico, } \\
\text { resultados clínicos...) }\end{array}$ & $\begin{array}{l}\text { Evaluaciones externas } \\
\text { (pacientes, colegas...) }\end{array}$ \\
\hline Resultados exámenes & Casos clínicos \\
\hline Publicaciones (artículos, libros,...) & Proyectos de Investigación \\
\hline Proyectos comunitarios & Premios y logros personales \\
& o profesionales \\
\hline Imaginación, creatividad... & \\
\hline
\end{tabular}

En esta línea, semFYC crea en Nov-2004 un grupo de trabajo para el desarrollo de un portafolio del médico de familia que pueda aportar un feed-back y ayudar al desarrollo de los profesionales a la vez que engarza esa información con la planificación de actividades informativas (una vez identificadas a nivel colectivo), acreditación de actividades...

Para ello se han seguido los siguientes pasos:

1.- Definición de bases estratégicas. La herramienta que se diseña está dirigida a médicos de familia que se encuentran en centros de salud de cualquier ámbito (urbanos o rurales, docentes o no), enfocado principalmente en esta fase a la autoevaluación, sin tutor presencial inicialmente, con un coste no excesivo en tiempo de dedicación del profesional evaluado (dada la presión asistencial existente) y con periodos adecuados de tiempo (entre 6 y 9 meses de desarrollo), que 
busque la globalidad con un nivel de calidad básico, con la Web como elemento de intercambio de información y tutorización online, y que permita en una segunda fase la posibilidad de realizar un portafolio evaluativo para recertificación o acreditación.

2.- Diseño del perfil del Medico de familia tanto de áreas competenciales como de competencias nucleares mas importantes para lo que se ha implicado a 23 profesionales relevantes entre presidentes de la Comisión Nacional de la especialidad, presidentes, directivos y asesores de semfyc y un representante clínico de prestigio de cada Sociedad autonómica. Como novedad se ha contado con la opinión de Asociaciones integradas en Foro Español de Pacientes y encuestas a pacientes claves de cupos de diferentes autonomías (32 en total).
Así se ha obtenido un Mapa de Competencias con 26 competencias operativas en las que se ha incluido también la valoración de expertos en evaluación del Grupo de desarrollo del portafolio priorizando aquellas que van a ser utilizadas en el Portafolio semFYC.

3.- Diseño de Indicadores, Tareas clínicas a realizar e indicadores operativos que el propio profesional va a buscar en los documentos que genere para autoevaluar su nivel de calidad en cada una de las competencias del Mapa.

4.- Diseño de la Web y aplicación que servirá de soporte para el desarrollo del portafolio, base de datos... con los sistemas de seguridad y confidencialidad necesarios. Incluye feed-back automático tras la evaluación final asi como ayudas a la formación en cada una de las competencias o áreas. 